\title{
SAPONINAS TRITERPÊNICAS DE Tocoyena brasiliensis MART. (RUBIACEAE)
}

Lidilhone Hamerski, Carlos Alberto Carbonezi, Alberto José Cavalheiro e Vanderlan da Silva Bolzani*

Instituto de Química, Universidade Estadual Paulista "Júlio de Mesquita Filho", CP 355, 14801-970 Araraquara - SP

Maria Cláudia Marx Young

Seção de Fisiologia e Bioquímica de Plantas, Instituto de Botânica, CP 4005, 01061-970 São Paulo - SP

Recebido em 19/4/04; aceito em 15/12/04; publicado na web em 13/4/05

TRITERPENOID SAPONINS FROM Tocoyena brasiliensis MART. (RUBIACEAE). The present communication reports the isolation and identification of four triterpenoid saponins from the chloroform extract of the leaves of Tocoyena brasiliensis: $3-O-\beta$-Dquinovopyranosyl quinovic acid, 3- $O$ - $\beta$-D-quinovopyranosyl cincholic acid, 3-O- $\beta$-D-glucopyranosyl quinovic acid and the 28$O-\beta$-D-glucopyranosyl ester derivative of quinovic acid as binary mixtures, respectively. From the ethanol extract a flavonoid identified as ramnazin-3-O-rutinoside was obtained. The structures of these compounds were assigned by data analysis of $1 \mathrm{D}$ and 2D NMR spectrometry and comparison with data recorded in the literature for these compounds.

Keywords: Tocoyena brasiliensis; saponins; antifungal.

\section{INTRODUÇÃO}

O gênero Tocoyena (Rubiaceae), com cerca de 30 espécies, ocorre nas regiões pan e neotropical ${ }^{1}$. São espécies típicas do Cerrado, sendo encontradas preferencialmente no Cerrado stricto senso, mas também ocorrem em florestas com clima quente e úmido, como a Floresta Amazônica ${ }^{2}$. Todos os representantes de Tocoyena são árvores de pequeno porte ou arbustos. Este gênero pertence à subfamília Ixoroideae, tribo Gardenieae-Gardeniineae, sendo os iridóides considerados seu principal marcador quimiotaxonômico ${ }^{3}$. Investigações fitoquímicas previamente realizadas por nosso grupo sobre Rubiaceae, em particular T. formosa, conduziu ao isolamento de iridóides glicosilados e não glicosilados, uma saponina triterpênica derivada do ácido quinóvico e um flavanol glicosilado ${ }^{4,5}$. Além de iridóides, a subfamília é conhecida por metabolizar flavonóides, triterpenos, derivados fenólicos e alcalóides do tipo emetínicos, quinolínicos e poliindolenínicos ${ }^{1}$.

Tocoyena brasiliensis Mart., conhecida popularmente como genipapinho $^{6}$, não apresenta registro de trabalhos sobre sua composição química. Ensaios preliminares, objetivando a detecção de substâncias com atividade antifúngica, indicaram para o extrato clorofórmico das folhas desta espécie atividade fraca contra o fungo filamentoso Cladosporium cladosporioides. O presente trabalho descreve o isolamento e identificação de duas misturas binárias de saponinas triterpênicas e um flavonóide glicosilado. As misturas das saponinas $\mathbf{1 + 2}$ e $\mathbf{3 + 4}$ apresentaram atividade moderada contra o fungo $C$. cladosporioides. As estruturas das substâncias foram estabelecidas com base na análise dos dados de RMN 1D e 2D.

\section{RESULTADOS E DISCUSSÃO}

O estudo fitoquímico das folhas de $T$. brasiliensis resultou na separação de duas misturas binárias de saponinas triterpênicas, a primeira contendo o ácido 3-O- $\beta$-D-quinovopiranosídeo-quinóvico ${ }^{7}$ (1) e o ácido-3- $O$ - $\beta$-D-quinovopiranosídeo-cinchólico (2) e a segunda, o ácido 3-O- $\beta$-D-glucopiranosídeo-quinóvico ${ }^{8}$ (3) e o ácido 28-O- $\beta$-D-glucopiranosídeo quinóvico ${ }^{9}$ (4). A saponina identificada

*e-mail: bolzaniv@iq.unesp.br
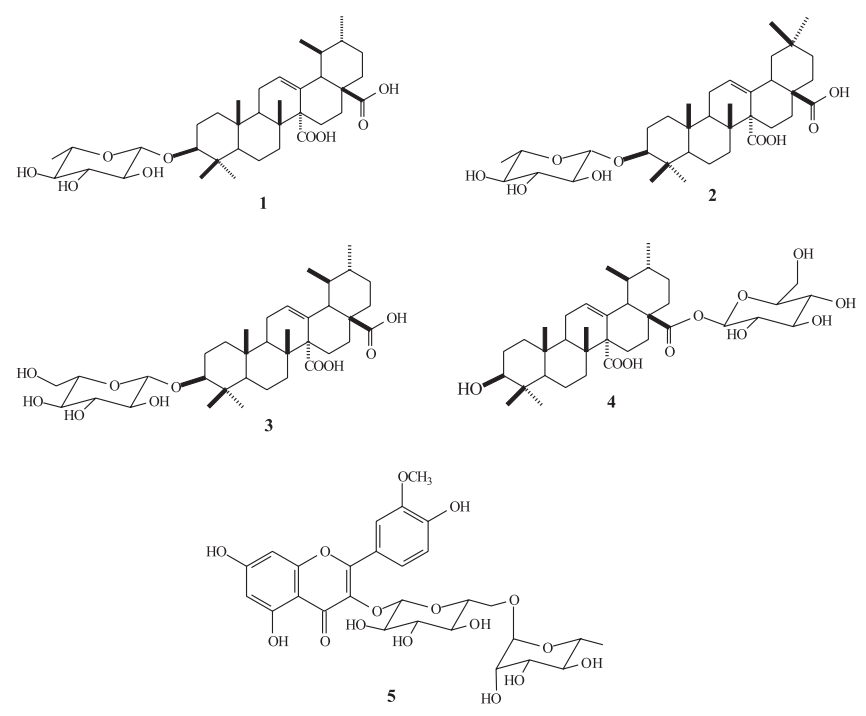

como o ácido 3-O- $\beta$-D-glucopiranosídeo-quinóvico ${ }^{8}(3)$ e o flavonóide ramanzina-3-O-rutinosídeo ${ }^{10}$ (5) também foram isolados como substâncias puras neste estudo.

As estruturas dos compostos isolados foram caracterizadas a partir da análise dos dados espectroscópicos (RMN de ${ }^{1} \mathrm{H} \mathrm{e}{ }^{13} \mathrm{C}$, DEPT 135, COSY ${ }^{1} \mathrm{H}^{1}{ }^{1} \mathrm{H}, g \mathrm{HMQC}, g \mathrm{HMBC}$, TOCSY 1D) e por comparação com os dados disponíveis na literatura.

As quatro saponinas triterpênicas isoladas apresentaram como padrão comum os C-27 e C-28 oxidados como grupo carboxílico. A ocorrência do grupo carboxila no C-27 foi evidenciada principalmente pela diferença $\Delta \delta$ no $\mathrm{C}-14$ de aproximadamente $+13,0$ ppm, quando comparado com a $\alpha$-amirina $(\mathrm{C}-14 \approx \delta 42,0)^{11}$. A diferença de deslocamento químico deste carbono é decorrente do efeito $\alpha$ da carboxila (C-27) em C-14. Assim, os esqueletos triterpênicos das saponinas isoladas foram identificados como derivados do ácido quinóvico ${ }^{12}$ ou do ácido cinchólico ${ }^{12}$, bastante discutidos e analisados na literatura.

A ocorrência da mistura binária das saponinas $\mathbf{1 + 2}$ foi evidenciada no espectro de $\mathrm{RMN}$ de ${ }^{1} \mathrm{H}$ pelos sinais em $\delta 5,49$ (m) e 
5,45 (m) atribuídos aos átomos de hidrogênio olefínicos (H-12). O espectro de $\mathrm{RMN}$ de ${ }^{13} \mathrm{C}$ mostrou sinais para dois esqueletos triterpênicos, sendo estes sinais análogos aos observados para ácido quinóvico ${ }^{12}$ e ácido cinchólico ${ }^{12}$. No espectro de RMN de ${ }^{13} \mathrm{C}$ foram observados os sinais em $\delta$ 128,1 (C-12), 132,8 (C-13), 55,5 (C-14), 53,8 (C-18), 38,6 (C-19), 36,2 (C-20), 176,9 (C-27), 178,8 (C-28), 18,2 (C-29) e 21,2 (C-30), que caracterizaram o esqueleto triterpênico do ácido quinóvico. Para o ácido cinchólico foram observados no espectro de $\mathrm{RMN}$ de ${ }^{13} \mathrm{C}$ os sinais em $\delta 125,1$ (C-12), 136,8 (C-13), 55,4 (C-14), 43,2 (C-18), 43,0 (C-19), 30,5 (C-20), 176,4 (C-27), 178,6 (C-28), 33,2 (C-29) e 23,5 (C-30) (Tabela 1). No espectro de $\mathrm{RMN}$ de ${ }^{1} \mathrm{H}$ pode-se identificar a presença de dois monossacarídeos por meio de seus átomos de hidrogênio anoméricos em $\delta 4,13(\mathrm{~d}, J=7,5)$ e 4,12 (d, $J=7,9)$, que foram correlacionados no mapa de contorno $g \mathrm{HMQC}$ aos átomos de carbono anoméricos em $\delta 105,2$ e 105,3, respectivamente. A presença de metila em $\delta 1,10(\mathrm{~d}, J=6,0)$ no espectro de RMN de ${ }^{1} \mathrm{H}$ e $\delta 18,1$ no espectro de $\mathrm{RMN}$ de ${ }^{13} \mathrm{C}$ sugeriu a presença de uma unidade de raminose, porém os valores dos deslocamentos químicos para os átomos de carbono não eram compatíveis aos observados para esse açúcar. No espectro de experimento COSY ${ }^{1} \mathrm{H}-{ }^{1} \mathrm{H}$ foram observadas as correlações entre os hidrogênios carbinólicos, que foram atribuídas inequivocamente aos seus respectivos carbonos pelo espectro de experimento $g$ HMQC. As constantes de acoplamento observadas para os H-1' $(J=7,5)$, H-2' $(J=9,0)$, H-3' $(J=9,0)$ e H-4' $(J=9,0)$ para ambas as unidades do açúcar indicaram a disposição diaxial dos átomos de hidrogênio do carboidrato (Tabela 2). Adicionalmente, o espectro de $g \mathrm{HMBC}$ apresentou correlações entre o H-6' $(\delta 1,10 \mathrm{~d}, J=6,0)$ com os C-5' $(\delta 75,5)$ e C-4' $(\delta 71,3)$; o H2' ( $\delta 2,94 \mathrm{~m})$ com o C-1'( $\delta 105,2)$. A associação destes dados aliados aos obtidos da literatura permitiram identificar a unidade de açúcar como a $\beta$-D-quinovose. A posição da unidade do açúcar no C-3 foi determinada pela correlação a longa distância, observada no mapa de contorno gHMBC entre o H-1' e o C-3 $(\delta 88,1)$. Após análise dos espectros de RMN e comparação com os dados da literatura foi possível identificar a substância 1 como ácido 3-O- $\beta$-Dquinovopiranosídeo-quinóvico ${ }^{7}$ e a substância 2 como o ácido-3$O$ - $\beta$-D-quinovopiranosídeo-cinchólico, inédita na literatura.

$\mathrm{O}$ espectro de $\mathrm{RMN}$ de ${ }^{13} \mathrm{C}$ da substância $\mathbf{3}$ apresentou sinais que revelaram a presença de uma saponina. Foram observados no espectro de RMN de ${ }^{13} \mathrm{C}$ os sinais em $\delta 128,0,132,5,55,3,176,3$, 178,6 atribuidos aos C-12, C-13, C-14, C-27 e C-28, respectivamente, do esqueleto triterpênico. Além do sinal de carbono anomérico em $\delta$ 105,6 (C-1'), outros sinais referentes a uma unidade glicosídica foram observados. Os deslocamentos químicos destes e dos demais carbonos, com suas respectivas multiplicidades, apresentaram valores concordantes com os da literatura para o ácido 3-O- $\beta$-D-glucopiranosídeo-quinóvico ${ }^{8}$ (3) (Tabela 1).

$\mathrm{O}$ espectro de $\mathrm{RMN}$ de ${ }^{13} \mathrm{C}$ da mistura $\mathbf{3}+\mathbf{4}$ apresentou sinais que indicaram se tratar de uma mistura binária de saponinas triterpênicas. Após identificação dos sinais atribuídos para a substância 3 no espectro de $\mathrm{RMN}$ de ${ }^{13} \mathrm{C}$, observaram-se os sinais para um segundo ácido quinóvico ${ }^{12}$, caracterizado principalmente pelos sinais duplicados em $\delta 55,3$ e 56,2; 53,7 e 53,8 atribuídos aos C-14 (3), C-14 (4), C-18 (3) e C-18 (4), respectivamente. A diferença entre as duas substâncias foi evidenciada pelos valores dos deslocamentos químicos dos átomos de carbono das unidades de açúcares. O valor do deslocamento químico do C-1' (substância 4) em $\delta$ 94,0 é observado quando a glicose está ligada ao C-28, conseqüência do efeito de blindagem da carboxila (Tabela 1). O espectro de experimento TOCSY 1D obtido pela irradiação no H-1' $(\delta 4,13$, d, $J=7,5)$ da glicose exibiu correlações com os sinais em $\delta 2,93$, $3,02,3,05$ e 3,10 atribuídos respectivamente aos H-2', H-4', H-5' e
H-3' da unidade glicosídica ligada ao C-3, da substância 3. A irradiação no H-1' ( $\delta 5,21$, d, $J=7,9)$ gerou um espectro de experimento TOCSY 1D onde foram observadas as correlações com os sinais em $\delta$ 2,89, 2,99 e 3,12, atribuídos aos H-4', H-2' e H-3', respectivamente, pertencentes à glicose ligada ao C-28 da substância 4. Os dados obtidos com o espectro de experimento TOCSY 1D aliados aos espectros de $g$ HMQC e $g$ HMBC auxiliaram na atribuição inequívoca das unidades de glicose. A análise dos dados de RMN 1D e 2D e comparação com os descritos na literatura permitiram identificar a substância 4 como ácido 28-O- $\beta$-D-glucopiranosídeo quinóvico9.

O flavonóide ramanzina-3-O-rutinosídeo (5) foi identificado pela comparação de seus dados espectroscópicos de RMN de ${ }^{1} \mathrm{H}$ e ${ }^{13} \mathrm{C}$ com os registrados na literatura ${ }^{10}$. A posição da unidade de $\beta$ D-glicose no C-3 foi confirmada pela correlação observada no espectro de $g \mathrm{HMBC}$ entre o $\mathrm{H}-1$ " $(\delta 5,38, \mathrm{~d}, J=8,0)$ e o C-3 $(\delta$ 133,5). A posição da metoxila no C-3' do anel B do flavonóide foi confirmada pela correlação observada entre os átomos de hidrogênio da metoxila $(\delta 3,83$, s) e o C-3' $(\delta 147,0)$.

A atividade antifúngica detectada na fase clorofórmica das folhas de $T$. brasiliensis contra o fungo fitopatogênico $C$. cladosporioides pode ser atribuída à presença de saponinas

Tabela 1. Dados de RMN de ${ }^{13} \mathrm{C}$ de 1, 2, 3 e 4 (DMSO- $d_{6}, 125$ $\mathrm{MHz}$

\begin{tabular}{|c|c|c|c|c|}
\hline $\mathrm{C}$ & 1 & 2 & 3 & 4 \\
\hline 1 & $38,8(\mathrm{t})$ & $38,8(\mathrm{t})$ & $38,6(\mathrm{t})$ & $39,0(\mathrm{t})$ \\
\hline 2 & $24,2(\mathrm{t})$ & $25,1(\mathrm{t})$ & $24,1(\mathrm{t})$ & $24,4(\mathrm{t})$ \\
\hline 3 & 88,1 (d) & 88,1 (d) & $88,0(\mathrm{~d})$ & $77,6(d)$ \\
\hline 4 & $39,0(\mathrm{~s})$ & $39,0(\mathrm{~s})$ & $39,0(\mathrm{~s})$ & $38,7(\mathrm{~s})$ \\
\hline 5 & 55,3 (d) & 55,3 (d) & 55,3 (d) & $54,8(d)$ \\
\hline 6 & $17,6(t)$ & 17,9 (t) & $17,8(\mathrm{t})$ & $17,7(\mathrm{t})$ \\
\hline 7 & $36,7(\mathrm{t})$ & $33,5(\mathrm{t})$ & $36,3(\mathrm{t})$ & $36,1(\mathrm{t})$ \\
\hline 8 & $39,0(\mathrm{~s})$ & $39,0(\mathrm{~s})$ & 38,9 (s) & $38,9(\mathrm{~s})$ \\
\hline 9 & 46,3 (d) & 46,6 (d) & 46,2 (d) & 46,8 (d) \\
\hline 10 & 38,5 (s) & 38,5 (s) & 38,7 (s) & $38,7(\mathrm{~s})$ \\
\hline 11 & $22,3(\mathrm{t})$ & $22,6(\mathrm{t})$ & $22,4(\mathrm{t})$ & $23,5(\mathrm{t})$ \\
\hline 12 & $128,1(\mathrm{~d})$ & $125,1(\mathrm{~d})$ & $128,0(\mathrm{~d})$ & $128,0(\mathrm{~d})$ \\
\hline 13 & $132,8(\mathrm{~s})$ & $136,8(\mathrm{~s})$ & $132,5(\mathrm{~s})$ & $132,6(\mathrm{~s})$ \\
\hline 14 & $55,5(\mathrm{~s})$ & $55,4(\mathrm{~s})$ & $55,3(\mathrm{~s})$ & $56,2(\mathrm{~s})$ \\
\hline 15 & $29,2(\mathrm{t})$ & $30,5(\mathrm{t})$ & $29,8(\mathrm{t})$ & $29,1(\mathrm{t})$ \\
\hline 16 & $25,9(\mathrm{t})$ & $24,0(\mathrm{t})$ & $25,0(\mathrm{t})$ & $25,7(\mathrm{t})$ \\
\hline 17 & $46,5(\mathrm{~s})$ & $47,5(\mathrm{~s})$ & 47,3 (s) & $47,8(\mathrm{~s})$ \\
\hline 18 & 53,8 (d) & 43,2 (d) & 53,7 (d) & $53,8(d)$ \\
\hline 19 & 38,6 (d) & $43,0(\mathrm{t})$ & 38,5 (d) & $38,6(d)$ \\
\hline 20 & 36,2 (d) & $30,5(\mathrm{~s})$ & 36,6 (d) & 36,4 (d) \\
\hline 21 & $28,9(\mathrm{t})$ & $36,4(\mathrm{t})$ & $25,6(t)$ & $30,2(\mathrm{t})$ \\
\hline 22 & $36,7(\mathrm{t})$ & $31,5(\mathrm{t})$ & $35,9(\mathrm{t})$ & $37,0(\mathrm{t})$ \\
\hline 23 & 27,8 (q) & 27,8 (q) & 27,8 (q) & $28,2(q)$ \\
\hline 24 & 16,7 (q) & 16,6 (q) & $16,5(q)$ & $16,5(\mathrm{q})$ \\
\hline 25 & 16,1 (q) & 16,2 (q) & $16,1(q)$ & $16,1(q)$ \\
\hline 26 & $18,1(\mathrm{q})$ & $18,1(\mathrm{q})$ & $18,2(\mathrm{q})$ & 17,9 (q) \\
\hline 27 & $176,9(\mathrm{~s})$ & $176,4(\mathrm{~s})$ & $176,3(\mathrm{~s})$ & 175,3 (s) \\
\hline 28 & $178,8(\mathrm{~s})$ & $178,6(\mathrm{~s})$ & $178,6(\mathrm{~s})$ & $176,9(\mathrm{~s})$ \\
\hline 29 & $18,2(\mathrm{q})$ & $33,2(q)$ & $17,5(\mathrm{q})$ & $18,6(q)$ \\
\hline 30 & $21,2(q)$ & $23,5(\mathrm{q})$ & $21,1(\mathrm{q})$ & $21,2(\mathrm{q})$ \\
\hline $1^{\prime}$ & 105,2 (d) & $105,3(d)$ & $105,6(d)$ & 94,0 (d) \\
\hline $2^{\prime}$ & 74,3 (d) & 74,3 (d) & 74,0 (d) & $72,4(d)$ \\
\hline 3' & 76,6 (d) & 76,6 (d) & 76,9 (d) & $76,6(d)$ \\
\hline $4^{\prime}$ & 71,3 (d) & 71,3 (d) & 70,3 (d) & $69,6(d)$ \\
\hline 5, & 75,5 (d) & 75,5 (d) & 76,6 (d) & 76,6 (d) \\
\hline 6 & $18,1(q)$ & $18,1(q)$ & $61,3(\mathrm{t})$ & $60,8(\mathrm{t})$ \\
\hline
\end{tabular}


Tabela 2. Dados de RMN de ${ }^{1} \mathrm{H}$ dos açúcares das substâncias 1, 2, 3 e 4 (DMSO- $d_{6}, 500 \mathrm{MHz}$ )

\begin{tabular}{ccccc}
\hline Posição & $\mathbf{1}$ & $\mathbf{2}$ & $\mathbf{3}$ & $\mathbf{4}$ \\
\hline $1^{\prime}$ & $4,13(\mathrm{~d}, J=7,5)$ & $4,12(\mathrm{~d}, J=7,9)$ & $4,13(\mathrm{~d}, J=7,5)$ & $5,21(\mathrm{~d}, J=7,9)$ \\
$2^{\prime}$ & $2,94(\mathrm{t}, J=9,0)$ & $2,95(\mathrm{t}, J=9,0)$ & $2,93(\mathrm{t}, J=8,5)$ & $2,99(\mathrm{t}, J=9,0)$ \\
$3^{\prime}$ & $3,06(\mathrm{t}, J=9,0)$ & $3,06(\mathrm{t}, J=9,0)$ & $3,10(\mathrm{t}, J=8,5)$ & $3,12(\mathrm{t}, J=9,0)$ \\
$4^{\prime}$ & $2,77(\mathrm{t}, J=9,0)$ & $2,77(\mathrm{t}, J=9,0)$ & $3,02(\mathrm{t}, J=8,5)$ & $2,89(\mathrm{t}, J=9,0)$ \\
$5^{\prime}$ & $3,13 \mathrm{~m}$ & $3,13 \mathrm{~m}$ & $3,05 \mathrm{~m}$ & $3,07 \mathrm{~m}$ \\
$6^{\prime}$ & $1,10(\mathrm{~d}, J=6,0)$ & $1,10(\mathrm{~d}, J=6,0)$ & $3,62(\mathrm{~d}, J=11,0)$ & $3,65(\mathrm{~d}, J=11,0)$ \\
& & & $3,40(\mathrm{dd}, J=11,0 ; 4,0)$ & $3,38(\mathrm{~d}, J=11,0)$ \\
\hline
\end{tabular}

triterpênicas. As substâncias isoladas e identificadas foram submetidas a novos ensaios antifúngicos, utilizando a técnica da bioautografia $^{13}$, para se determinar os limites de detecção (quantidade mínima da amostra, em $\mu \mathrm{g}$, necessária para inibir o crescimento do fungo), sendo utilizados os fungos filamentosos $C$. cladosporioides e $C$. sphaerospermum (Tabela 3). As misturas $(\mathbf{1}+\mathbf{2})$ e $(\mathbf{3}+\mathbf{4})$ apresentaram atividade moderada $(5 \mu \mathrm{g} / \mathrm{mL})$ contra $C$. cladosporioides em comparação com o padrão nistatina $(0,5 \mu \mathrm{g} / \mathrm{mL})$ e não inibiram o crescimento de $C$. sphaerospermum. Não foi observada atividade antifúngica para a substância $\mathbf{3}$, este resultado é um indício de que a atividade antifúngica observada para a mistura das saponinas $\mathbf{3}+\mathbf{4}$ pode ser atribuída para a saponina 4.

Tabela 3. Limite de detecção da atividade antifúngica em $\mu \mathrm{g} / \mathrm{mL}$

\begin{tabular}{ccc}
\hline Substâncias & C. cladosporioides & C. sphaerospermum \\
\hline $1+2$ & 5 & $\mathrm{i}$ \\
3 & $\mathrm{i}$ & $\mathrm{i}$ \\
$3+4$ & 5 & $\mathrm{i}$ \\
5 & $*$ & $*$ \\
nistatina & 0,5 & 0,5 \\
\hline
\end{tabular}

i - inativo, *- não testado

\section{PARTE EXPERIMENTAL}

\section{Procedimentos experimentais gerais}

Os espectros de RMN foram registrados em DMSO- $d_{6}$, no espectrômetro Bruker AC-200 F e Varian Inova 500, usando TMS como padrão de referência. Os experimentos TOCSY, COSY, $g \mathrm{HMBC}$ e $g \mathrm{HMQC}$ foram realizados em espectrômetro Varian Inova 500 e DEPT $135^{\circ}$ em espectrômetro Bruker AC-200 F. Os espectros de massas foram registrados em espectrômetro de massas de baixa resolução FISION Modelo VG Platfform II, no modo Eletrospray. Os espectros no IR foram registrados em espectrofotômetro Perkin-Elmer 1600 (Series FTIR), em células de KBr. Os espectros no UV foram registrados em espectrofotômetro PerkinElmer Lambda 14P UV/VIS. Nas análises por CCDC e CCDP foram utilizadas placas de sílica gel $60 \mathrm{G} \mathrm{F} 254$. As placas foram observadas sob luz UV 254-366 nm (Chromatovus) e reveladas com vapores de iodo ressublimado e solução de anisaldeído, seguida de aquecimento. Nas cromatografias em coluna foram utilizadas como fase estacionária sílica gel (230-400 mesh), sílica quimicamente modificada com grupos octadecila (ODS) (Merk) e Sephadex LH-20.

\section{Material vegetal}

As folhas de T. brasiliensis foram coletadas na Estação Ecológica de Itirapina, Itirapina - SP em janeiro de 2000. Uma exsicata encontra-se depositada no Herbário do Instituto de Botânica da Secretaria do Meio Ambiente de São Paulo.

\section{Extração e isolamento dos constituintes}

As folhas de T. brasiliensis (2.400 g) foram secas, moídas e submetidas à extração através de maceração com EtOH/ $\mathrm{H}_{2} \mathrm{O}(8: 2)$. $\mathrm{O}$ extrato $\mathrm{EtOH} / \mathrm{H}_{2} \mathrm{O}$ foi concentrado sobre pressão reduzida obtendo-se 210,0 g de extrato bruto, sendo 130,0 g submetidos à partição utilizando $n-\mathrm{BuOH} / \mathrm{H}_{2} \mathrm{O}(1: 1)$. A fase $n-\mathrm{BuOH}$ foi concentrada à pressão reduzida, novamente solubilizada em $\mathrm{MeOH} / \mathrm{H}_{2} \mathrm{O}(9: 1)$ e, em seguida, submetida à partição com hexano (48,3 g), clorofórmio $(11,2 \mathrm{~g})$ e acetato de etila $(11,2 \mathrm{~g})$.

Uma fração da fase $\mathrm{CHCl}_{3}(4,9 \mathrm{~g})$ das folhas foi submetida à cromatografia líquida a vácuo utilizando sílica gel como fase estacionária, eluída em gradiente de polaridade [Hex; $\mathrm{Hex} / \mathrm{CH}_{2} \mathrm{Cl}_{2}(8: 2)$ $\rightarrow$ (4:6); $\mathrm{CH}_{2} \mathrm{Cl}_{2} ; \mathrm{CH}_{2} \mathrm{Cl}_{2} / \mathrm{MeOH}$ (95:5) $\rightarrow$ (6:4); $\left.\mathrm{MeOH}\right]$, da qual foram coletadas 18 frações (70 mL cada). A fração 11 (1,09 g) foi fracionada em coluna de sílica gel eluída com $\mathrm{CH}_{2} \mathrm{Cl}_{2} / \mathrm{MeOH}$ (9:1), obtendo-se a mistura das saponinas $\mathbf{1 + 2}(32,4 \mathrm{mg})$ e a substância 3 (27,0 mg). A subfração 11-B (176,6 mg), oriunda da fração 11 , foi submetida a fracionamento em coluna de sílica gel, eluída com $\mathrm{CH}_{2} \mathrm{Cl}_{2} / \mathrm{MeOH}$ (8:2), obtendo a mistura das saponinas $\mathbf{3 + 4}(20,8$ $\mathrm{mg})$. O extrato etanólico das folhas $(10,0 \mathrm{~g})$ foi solubilizado em $\mathrm{EtOH} / \mathrm{H}_{2} \mathrm{O}(6: 4)$ e submetido à cromatografia líquida a vácuo utilizando sílica de fase modificada (C-18) como fase estacionária e eluída em gradiente de polaridade $\left[\mathrm{MeOH} / \mathrm{H}_{2} \mathrm{O}(2: 8) \rightarrow(6: 4)\right.$, $\mathrm{MeOH}, \mathrm{CH}_{2} \mathrm{Cl}_{2}$ ] sendo obtidas 8 frações (100 mL cada). A fração 6 foi submetida a fracionamento em Sephadex LH-20, eluída com $\mathrm{MeOH}$, obtendo-se o flavonóide glicosilado 5 (32,5 mg).

Ácido 3-O- $\beta$-D-quinovopiranosídeo-quinóvico (1)

Sólido amorfo branco. RMN ${ }^{1} \mathrm{H} \delta\left(500 \mathrm{MHz}, \mathrm{DMSO}-d_{6}\right): 2,95$ $(1 \mathrm{H}, \mathrm{m}, \mathrm{H}-3), 0,50$ (1H, m, H-5), 5,45 (1H, m, H-12), 0,90 (3H, s, $\mathrm{H}-23), 0,70$ (3H, s, H-24), 0,83, (3H, s, H-25), 0,73 (3H, s, H-26), $0,75(3 \mathrm{H}, \mathrm{d}, J=4,5, \mathrm{H}-29), 0,85$ (3H, d, $J=5,0, \mathrm{H}-30)$; $\beta$-Dquinovose: ver Tabela $2 . \mathrm{RMN}{ }^{13} \mathrm{C}$ : ver Tabela 1.

Ácido-3-O- $\beta$-D-quinovopiranosídeo-cinchólico (2)

Sólido amorfo branco. RMN ${ }^{1} \mathrm{H} \delta$ (500 MHz, DMSO- $\left.d_{6}\right):$ 2,95 $(1 \mathrm{H}, \mathrm{m}, \mathrm{H}-3), 0,50(1 \mathrm{H}, \mathrm{m}, \mathrm{H}-5), 5,49(1 \mathrm{H}, \mathrm{m}, \mathrm{H}-12), 0,90(3 \mathrm{H}, \mathrm{s}$, H-23), 0,70 (3H, s, H-24), 0,83, (3H, s, H-25), 0,73 (3H, s, H-26), $0,79$ (3H, d, $J=4,5, \mathrm{H}-29), 0,84$ (3H, d, $J=5,0, \mathrm{H}-30)$; $\beta-\mathrm{D}-$ quinovose: ver Tabela 2 . RMN ${ }^{13} \mathrm{C}$ : ver Tabela 1.

Ácido 3-O- $\beta$-D-glucopiranosídeo-quinóvico (3)

Sólido amorfo branco. IR $v_{\max } \mathrm{cm}^{-1}: 3400,2935,1698,1550$, $1446,1390,1310,1225,1057,980$. RMN ${ }^{1} \mathrm{H} \delta$ (500 MHz, DMSO$\left.d_{6}\right): 1,57(2 \mathrm{H}, \mathrm{m}, \mathrm{H}-1), 1,55(2 \mathrm{H}, \mathrm{m}, \mathrm{H}-2), 2,98(1 \mathrm{H}, \mathrm{m}, \mathrm{H}-3), 0,58$ $(1 \mathrm{H}, \mathrm{d}, J=12,0, \mathrm{H}-5), 1,90$ (2H, m, H-11) 5,46 (1H, m, H-12), 1,36 (2H, m, H-15), 1,52 (2H, m, H-16), 2,12 (1H, d, $J=10,0, \mathrm{H}-$ 
18), 1,51 (2H, m, H-20), 1,89 (2H, m, H-21), 0,92 (3H, s, H-23), 0,71 (3H, s, H-24), 0,86, (3H, s, H-25), 0,76 (3H, s, H-26), 0,84 (3H, d, $J=5,0, \mathrm{H}-29), 0,86$ (3H, d, $J=5,0, \mathrm{H}-30)$; $\beta$-D-glicose: ver Tabela 2. RMN ${ }^{13} \mathrm{C}$ : ver Tabela 1.

Ácido 28-O- $\beta$-D-glucopiranosídeo quinóvico (4)

Sólido amorfo branco. RMN ${ }^{1} \mathrm{H} \delta$ (500 MHz, DMSO- $\left.d_{6}\right): 2,93$ (1H, m, H-3), 0,52 (1H, m, H-5), 5,45 (1H, m, H-12), 0,92 (3H, s, H-23), 0,74 (3H, s, H-24), 0,83, (3H, s, H-25), 0,73 (3H, s, H-26), $0,79$ (3H, d, $J=4,5, \mathrm{H}-29), 0,84$ (3H, d, $J=4,5, \mathrm{H}-30$ ); $\beta$-D-glicose: ver Tabela 2. RMN ${ }^{13} \mathrm{C}$ : ver Tabela 1.

\section{Ramanzina-3-O-rutinosídeo (5)}

Sólido amorfo amarelo. UV (MeOH) $\lambda_{\text {máx }} \mathrm{nm:}$ 285, 342. EMES $(70 \mathrm{eV}), m / z, 651[\mathrm{M}-\mathrm{H}]^{-}$. RMN ${ }^{1} \mathrm{H} \delta\left(500 \mathrm{MHz}, \mathrm{DMSO}-d_{6}\right)$ : 6,04 (1H, sl, H-6), 6,25 (1H, sl, H-8), 7,97 (1H, d, $J=2,0, \mathrm{H}-2$ '), 6,87 ( $1 \mathrm{H}, \mathrm{d}, J=8,5, \mathrm{H}-5$ '), 7,45 (1H, dd, $J=2,0 ; 8,5, \mathrm{H}-6$ ') $, 3,83$ $\left(3 \mathrm{H}, \mathrm{s}, \mathrm{OC} \underline{\mathrm{H}}_{3}\right)$; $\beta$-D-glicose: 5,38 (1H, d, $J=8,0, \mathrm{H}-1$ "), 3,30 (1H, m, H-2"), 3,64 (1H, t, $J=8,5, \mathrm{H}-3$ "), 3,58 (1H, d, $J=8,5, \mathrm{H}-4$ "), 3,09 (1H, d, $J=8,5, \mathrm{H}-5$ ”), 3,30 (1H, m, H-6"), 3,62 (1H, m, H6."); $\alpha$-L-ramnose: 4,42 (1H, sl, H-1'”), 3,79 (1H, m, H-2'”), 3,38 (1H, dd, $J=3,0$; 9,0, H-3"'), 3,15 (1H, m, H-4"'), 3,75 (1H, m, H5 '”), 1,05 (3H, d, $J=6,0, \mathrm{H}-6$ '"). RMN ${ }^{13} \mathrm{C} \delta$ (125 MHz, DMSO$\left.d_{6}\right)$ : 156,8 (C-2), 133,5 (C-3), 177,7 (C-4), 160,8 (C-5), 99,3 (C-6), 161,7 (C-7), 94,7 (C-8), 156,0 (C-9), 105,6 (C-10), 120,9 (C-1'), 113,4 (C-2'), 147,0 (C-3'), 149,7 (C-4'), 115,2 (C-5'), 122,2 (C6'), 55,9 $\left(\mathrm{OCH}_{3}\right)$; $\beta$-D-glicose: 101,7 (C-1"), 74,1 (C-2”), 76,9 (C3"), 70,5 (C-4"), 76,5 (C-5"), 65,1 (C-6"); $\alpha$-L-ramnose: 100,0 (C1"'), 70,3 (C-2"'), 70,4 (C-3"'), 71,6 (C-4"'), 68,3 (C-5"'), 17,9 (C-6"').

\section{BIOAUTOGRAFIA}

O ensaio bioautográfico foi realizado de acordo com a técnica descrita por Homanns e Fuchs ${ }^{13}$, utilizando como reveladores os fungos filamentosos C. cladosporioides e C. sphaerospermum. Nistatina foi utilizada como padrão.

\section{AGRADECIMENTOS}

À FAPESP, programa Biota-FAPESP (Instituto Virtual da Biodiversidade - www.biota.org.br) e ao $\mathrm{CNPq}$, pelo apoio financeiro e pelas bolsas.

\section{REFERÊNCIAS}

1. Robbrecht, E. Em Advances in Rubiaceae Macrosystematic; Op. Bot. Belg., National Botanical Garden Publishers: Meise, Belgium, 1994, vol 6.

2. Gottsberger-Silberbauer, I.; Gottsberger, G.; Ehrendorfer, F. Pl.; Syst. Evol. 1992, 181, 131.

3. Robbrecht, E.; Puff, C.; Smets, E.; Second International Rubiaceae Conference, Op. Bot. Belg., National Botanical Garden Publishers: Meise, Belgium, 1996.

4. Bolzani, V. da S.; Trevisan, L. M. V.; Izumisawa, C. M.; Young, M. C. M.; J. Braz. Chem. Soc. 1996, 7, 157.

5. Bolzani, V. da S.; Trevisan, L. M. V.; Izumisawa, C. M.; Young, M. C. M.; Kingston, D. G. I.; Gunatilaka, A. A. L.; Phytochemistry 1997, 46, 305.

6. http://www.ibot.sp.gov.br/resolucao_sma47/anexo.pdf, acessada em Março 2004.

7. Yépes, A. M. P.; Ugaz, O. L.; Alvarez, C. M. P. A.; Feo, V.; Aquino, R.; Simone, F.; Pizza, C.; Phytochemistry 1991, 30, 1635.

8. Sousa, M. P.; Matos, M. E. O.; Machado, M. I. L.; Braz Filho, R.; Vecanto, I.; Mascarenhas, Y. P.; Phytochemistry 1984, 23, 2589.

9. Hassanean, H. H.; Desok, E. K.; El-Hamouly, M. M. A.; Phytochemistry 1993, 33, 663 .

10. Agrawal, P. K.; Carbon-13 NMR of flavonoids, Elsevier: New York, 1980.

11. Mahato, S.; Kundu, A.; Phytochemistry 1994, 37, 1516.

12. Humberto-Sanchez, A.; Vasquez, P.; Phytochemistry 1991, 30, 623.

13. Homanns, A. L.; Fuchs, A.; J. Chromatogr. 1970, 51, 327. 